\title{
Torsion of anisotropic and non-uniform cylindrical rods with elliptical section
}

\author{
Boris Mironov ${ }^{*}$ and Yuriy Mironov ${ }^{\dagger}$ \\ ${ }^{1}$ RUT, Department of Higher mathematics and Natural Sciences, 127994, Moscow, Chasovaya str., \\ h. 22, p. 2 \\ ${ }^{2}$ MTUCI, Research Department, 111024, Moscow, Aviamotornaya str., 8A
}

\begin{abstract}
In work the limit state of cylindrical and prismatic rods from anisotropic ideal rigid-plastic material is investigated under torsion for arbitrary condition of plasticity, and the torsion of anisotropic and nonuniform rods with elliptic section under the condition of Mises-Hill plasticity is considered. The integrals determining the stressed state of an anisotropic rod under arbitrary condition of plasticity are obtained, the field of characteristics of the basic ratios for anisotropic and composite rods under the condition of Mises-Hill plasticity is constructed, the ratios along characteristics are obtained, the envelopes of the family of characteristics and lines of tension rupture are found.
\end{abstract}

\section{Introduction}

Torsion is a type of deformation of solids characterized by mutual rotation of its cross sections under the influence of moments operating in these sections. Torsion of rods is quite common in engineering practice, especially in mechanical engineering. Shafts of engines and machines, axles of wagons and locomotives, helical springs, etc. work by torsion. The theory of torsion of isotropic and anisotropic rods belongs to the number of developed sections of the theory of an ideal rigid-plastic solid. At the same time, researches on the theory of torsion of non-uniform rods are not enough. In particular, researches on the theory of torsion of isotropic and anisotropic ideal rigid-plastic rods are presented in works [1]-[4]. Torsion of non-uniform and compound rods is considered in works [5]-[7]. The results obtained in the work can be applied in mechanical engineering in determining of ultimate loadings on the rod as a result of torsion, in solving of new problems of the theory of limit state, in calculations of the bearing capacity of various solids and structures.

\footnotetext{
*Corresponding author: mironov.boris.21@gmail.com

${ }^{\dagger}$ Corresponding author: mistiam@gmail.com
} 


\section{Methods and Results}

\subsection{Torsion of an anisotropic rod under arbitrary plasticity condition}

Consider a cylindrical or prismatic rigid-plastic rod oriented in a rectangular coordinate system $x y z$. Generatrices of the rod are parallel to the axis $z$. Assume that the rod consists of anisotropic ideal-plastic material. The rod is twisted around its axis, the lateral surface of the rod is free from loadings.

Let us assume that the stress state arising in the rod is characterized by following values of the tension components:

$$
\begin{gathered}
\sigma_{x}=\sigma_{y}=\sigma_{z}=\tau_{x y}=0, \\
\tau_{x z}=\tau_{x z}(x, y), \tau_{y z}=\tau_{y z}(x, y) .
\end{gathered}
$$

In general case, the plasticity condition has the form

$$
f\left(\tau_{x z}, \tau_{y z}\right)=0
$$

and the only equation of equilibrium takes the form

$$
\frac{\partial \tau_{x z}}{\partial x}+\frac{\partial \tau_{y z}}{\partial y}=0
$$

As it follows from our assumptions, the equality is carried out on the contour of cross section of the rod

$$
\frac{d y}{d x}=\frac{\tau_{y z}}{\tau_{x z}}
$$

Taking into account (2), the characteristics of equation (3) are determined by the ratio

$$
\frac{d y}{d x}=\frac{\frac{\partial f}{\partial \tau_{x z}}}{\frac{\partial f}{\partial \tau_{y z}}}
$$

and have the form

$$
p x+q y=\text { const }
$$

and along the characteristics we have the ratios

$$
\tau_{x z}=c_{1}=\text { const, } \tau_{y z}=c_{2}=\text { const }
$$

where $p=\frac{\partial f}{\partial \tau_{x z}}\left(c_{1}, c_{2}\right), q=\frac{\partial f}{\partial \tau_{y z}}\left(c_{1}, c_{2}\right), f\left(c_{1}, c_{2}\right)=0$.

Thus, according to (5), (6) and (7), characteristics of the ratio (3) are lines orthogonal to the gradient vector to the yield curve (2) at the corresponding point.

\subsection{Torsion of an anisotropic rod with elliptical section}

Consider an anisotropic cylindrical rod, which contour L of the cross section is an ellipse (Fig. 1a). 


$$
\mathrm{L}: \frac{x^{2}}{a_{0}^{2}}+\frac{y^{2}}{b_{0}^{2}}=1
$$

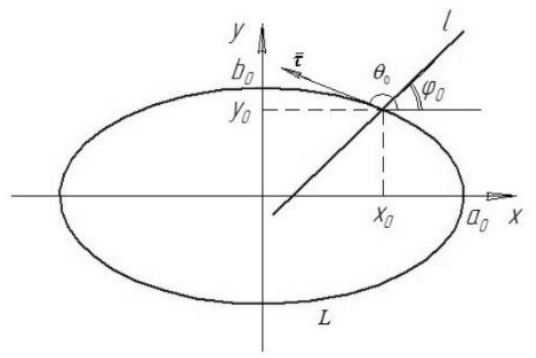

a)

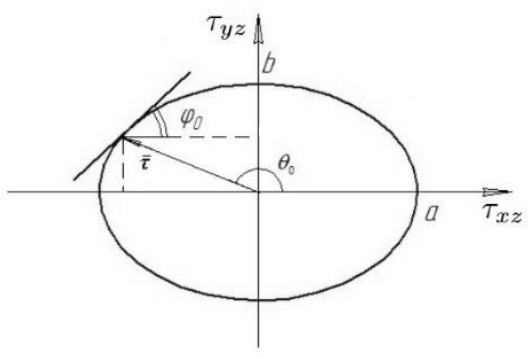

б)

Fig. 1. Section of an anisotropic rod with characteristic and yield curve

Assume that the plasticity condition (2) is given in the form (Fig. 1b)

$$
\frac{\tau_{x z}^{2}}{a^{2}}+\frac{\tau_{y z}^{2}}{b^{2}}=1
$$

Let the point $\left(x_{0}, y_{0}\right) \in L$, and the characteristic $l$ of the relation (2) passes through it. According to (6), (7), (8) and (9) the equation of the family of characteristics $l$ has the form

$$
a^{2} b_{0}^{2} x_{0}\left(y-y_{0}\right)-a_{0}^{2} b^{2} y_{0}\left(x-x_{0}\right)=0
$$

and tension components are given along the characteristics in the form

$$
\tau_{x z}=-\frac{a_{0}^{2} a b y_{0}}{\sqrt{a_{0}^{2} b^{2} y_{0}^{2}+b_{0}^{2} a^{2} x_{0}^{2}}}, \tau_{y z}=\frac{b_{0}^{2} a b x_{0}}{\sqrt{a_{0}^{2} b^{2} y_{0}^{2}+b_{0}^{2} a^{2} x_{0}^{2}}}
$$

The family of characteristics (10) has an envelope

$$
\left(a_{0} b^{2} x\right)^{\frac{2}{3}}+\left(b_{0} a^{2} y\right)^{\frac{2}{3}}=\left(a_{0}^{2} b^{2}-a^{2} b_{0}^{2}\right)^{\frac{2}{3}}
$$

In particular, if the equality $\frac{a}{b}=\frac{a_{0}}{b_{0}}$ is satisfied, then the ratios (10) and (11) take the form

$$
\begin{gathered}
y=\frac{y_{0}}{x_{0}} x, \\
\tau_{x z}=-\frac{a y_{0}}{b_{0}}, \tau_{y z}=\frac{b x_{0}}{a_{0}} .
\end{gathered}
$$

\subsection{Torsion of a non-uniform rod with elliptical section}

Consider a non-uniform cylindrical ideal-plastic rod. Section contour of the rod by the plane $z=$ const is determined by the ratio (8).

Assume that section of the rod consists of two anisotropic areas separated by AOB broken line (Fig. 2a, 2b). 


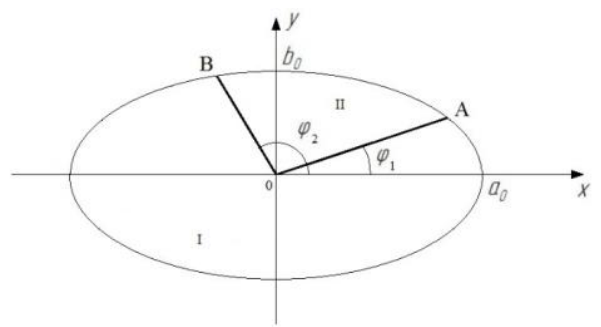

a) $0<\gamma<\pi$

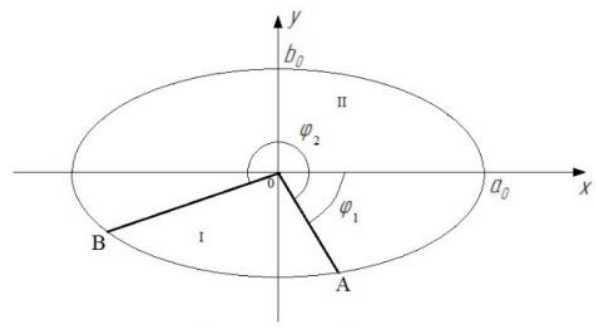

б) $\pi<\gamma<2 \pi$

Fig. 2. Section of a composite rod

The rod is twisted around the $z$ axis by equal and opposite pairs of forces. Lateral surface of the rod is considered free from loadings.

Stress state of the rod is determined by the ratios (1) and the plasticity conditions

$$
\frac{\tau_{x z}^{2}}{a_{1}^{2}}+\frac{\tau_{y z}^{2}}{b_{1}^{2}}=1
$$

in area I,

$$
\frac{\tau_{x z}^{2}}{a_{2}^{2}}+\frac{\tau_{y z}^{2}}{b_{2}^{2}}=1
$$

in area II, by equilibrium equation (3), where $b_{1} \leq b_{2}, a_{1} \leq a_{2}$.

According to (5), characteristics of the ratio (3) in each area are straight lines orthogonal to the gradient vector to the curves (15) and (16), respectively. The vector of tangent tension:

$$
\bar{\tau}=\tau_{x z} \bar{i}+\tau_{y z} \bar{j}
$$

does not change along the characteristics and is directed along the tangent to the contour (8) of cross-section of the rod.

Let

$$
\frac{b_{1}}{a_{1}}=\frac{b_{2}}{a_{2}}=\frac{b_{0}}{a_{0}}
$$

Then the equation of characteristics in both areas has the form (13).

According to (14), the vector of tangent tension $\bar{\tau}$ in areas I and II is respectively determined by the ratios

$$
\begin{aligned}
& \bar{\tau}=-\frac{a_{1}}{b_{0}} y_{0} \bar{i}+\frac{b_{1}}{a_{0}} x_{0} \bar{j}, \\
& \bar{\tau}=-\frac{a_{2}}{b_{0}} y_{0} \bar{i}+\frac{b_{2}}{a_{0}} x_{0} \bar{j}
\end{aligned}
$$

A jump of the tangent stresses is inevitable on the line of heterogeneity AOB. Therefore, vector of tangent tension $\bar{\tau}$, and accordingly characteristics of the ratio (4) change their direction when passing through the broken line AOB. This leads to additional lines of tension rupture in area II (Fig. 3a, 3b).

Let the point $A$ have coordinates $\left(x_{1}, y_{1}\right)$ and point $B$ has coordinates $\left(x_{2}, y_{2}\right)$. Then 


$$
\operatorname{tg} \varphi_{1}=\frac{y_{1}}{x_{1}}, \operatorname{tg} \varphi_{2}=\frac{y_{2}}{x_{2}}
$$

where $\varphi_{1}$ is an angle formed by the line of heterogeneity $O A$ with axis $O x, \varphi_{2}$ is an angle formed by the line of heterogeneity $O B$ with axis $O x$. Suppose that: a) $0<\gamma<\pi$, б) $\pi<$ $\gamma<2 \pi$.

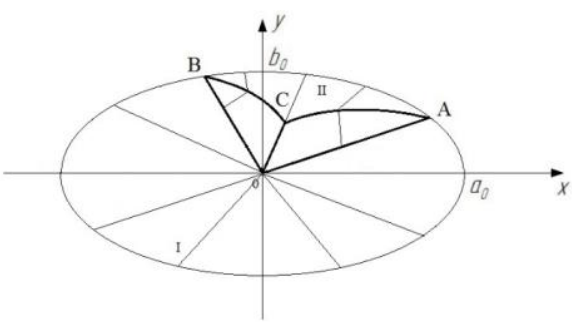

a) $0<\gamma<\pi$

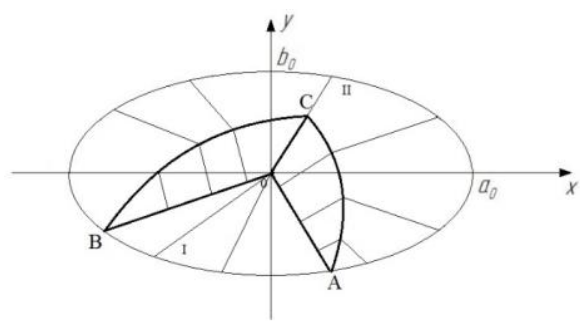

б) $\pi<\gamma<2 \pi$

Fig. 3. Field of characteristics and lines of tension rupture

Then, vector of tangent tension $\overrightarrow{\tau_{2}}$ in the area bounded by a closed curve OACO is determined by the ratio

$$
\overrightarrow{\tau_{2}^{\prime}}=\left(m x_{1}-\frac{a_{1}}{b_{0}} y_{1}\right) \vec{\imath}+\left(\frac{b_{1}}{a_{0}} x_{1}+m y_{1}\right) \vec{\jmath}
$$

where $m=\frac{\sqrt{a_{2}^{2}-a_{1}^{2}}}{a_{0}}=\frac{\sqrt{b_{2}^{2}-b_{1}^{2}}}{b_{0}}$.

Characteristics in this area are given by the equation

$$
b_{2}^{2}\left(m x_{1}-\frac{a_{1}}{b_{0}} y_{1}\right) x+a_{2}^{2}\left(\frac{b_{1}}{a_{0}} x_{1}+m y_{1}\right) y=\text { const }
$$

The line of tension rupture $A C$ is determined from the differential equation

$$
\left(\frac{b_{2} b_{0} x}{\sqrt{b_{0}^{2} x^{2}+a_{0}^{2} y^{2}}}-\left(\frac{b_{1}}{a_{0}} x_{1}+m y_{1}\right)\right) d x+\left(\frac{a_{2} a_{0} y}{\sqrt{b_{0}^{2} x^{2}+a_{0}^{2} y^{2}}}-\left(m x_{1}-\frac{a_{1}}{b_{0}} y_{1}\right)\right) d y=0 .
$$

According to (24), the equation of the line of tension rupture AC has the form

$$
\frac{b_{2}}{b_{0}} \sqrt{b_{0}^{2} x^{2}+a_{0}^{2} y^{2}}-\left(\frac{b_{1}}{a_{0}} x_{1}+m y_{1}\right) x+\left(m x_{1}-\frac{a_{1}}{b_{0}} y_{1}\right) y=a_{0}\left(b_{2}-b_{1}\right) \text {. }
$$

The vector of tangent tension $\overrightarrow{\tau_{2}^{\prime \prime}}$ in the area bounded by a closed curve $O B C O$ is determined by the ratio

$$
\overrightarrow{\tau_{2}^{\prime}}=\left(=m x_{2}-\frac{a_{1}}{b_{0}} y_{2}\right) \vec{\imath}+\left(\frac{b_{1}}{a_{0}}-m y_{2}\right) \vec{\jmath} .
$$

Characteristics in this area are given by the equation 


$$
b_{2}^{2}\left(-m x_{2}-\frac{a_{1}}{b_{0}} y_{2}\right) x+a_{2}^{2}\left(\frac{b_{1}}{a_{0}} x_{2}-m y_{2}\right) y=\text { const }
$$

The line of tension rupture $B C$ is determined from the differential equation

$$
\left(\frac{b_{2} b_{0} x}{\sqrt{b_{0}^{2} x^{2}+a_{0}^{2} y^{2}}}+\left(-\frac{b_{1}}{a_{0}} x_{2}+m y_{2}\right)\right) d x+\left(\frac{a_{2} a_{0} y}{\sqrt{b_{0}^{2} x^{2}+a_{0}^{2} y^{2}}}-\left(m x_{2}+\frac{a_{1}}{b_{0}} y_{2}\right)\right) d y=0
$$

According to (28), the equation of the line of tension rupture $B C$ has the form

$$
\frac{b_{2}}{b_{0}} \sqrt{b_{0}^{2} x^{2}+a_{0}^{2} y^{2}}+\left(-\frac{b_{1}}{a_{0}} x_{2}+m y_{2}\right) x-\left(m x_{2}+\frac{a_{1}}{b_{0}} y_{2}\right) y=a_{0}\left(b_{2}-b_{1}\right)
$$

\section{Conclusions}

Integrals describing the limit state of an anisotropic rigid-plastic rod at torsion for the arbitrary plasticity condition are obtained.

The stress state at torsion of an anisotropic rod with elliptic section for Mises-Hill plasticity condition is described: the field of characteristics of the basic ratios is constructed, the ratios along characteristics and the envelope of the family of characteristics of the basic ratios are found/

The limit state of a rigid-plastic composite rod with elliptical section is investigated: the field of characteristics of the basic ratios is constructed, the ratios along the characteristics and the lines of tension rupture are found.

\section{References}

1. G.I. Bykovtsev, Theory of plasticity (1998).

2. D.D. Ivlev, Theory of ideal plasticity (1966).

3. Ye.A. Derevyannyih, B.G. Mironov, Bulletin of the Yakovlev Chuvash State Pedagogical University, 4(76), 108-112 (2012).

4. B.G. Mironov, T.V. Mitrofanova, Bulletin of the Yakovlev Chuvash State Pedagogical University. Series: Mechanics of a limit state, 1(23), 197-200 (2015).

5. W. Olszak, J. Rychlewski, W. Urbanowski Plasticity under non-homogenous conditions (1964).

6. B.G. Mironov, Yu.B. Mironov, Bulletin of the Yakovlev Chuvash State Pedagogical University. Series: Mechanics of a limit state, 2(32), 18-22 (2017).

7. B.G. Mironov Bulletin of the Yakovlev Chuvash State Pedagogical University. Series: Mechanics of a limit state, 3(33), 95-100 (2017). 